\title{
Racing Under Uncertainty: A Boundary Value Problem Approach
}

\author{
Dan Cao* \\ Department of Economics, Georgetown University
}

October 25, 2010

\begin{abstract}
In this paper I formulate a continuous time and continuous space version of Harris and Vickers (1987) Racing Under Uncertainty with potentially asymmetric players. To prove the existence and uniqueness of the equilibria, I use a boundary value problem formulation which is novel to the dynamic competition literature. In some cases, I obtain closed-form solutions of the equilibria in which equilibrium strategies exhibit the discouragement effect similar to the one in the original paper.
\end{abstract}

*I wish to thank Daron Acemoglu for his advice. Also thanks to Axel Anderson, David Cesarini, JeanPaul L'Huillier, Giuseppe Moscarini, Yuliy Sannikov, Lones Smith, Ivan Werning and participants at the MIT Macro Lunch for helpful comments. 


\section{Introduction}

A race is a contest between two or more competitors who exert effort to win a prize. Sport contests, such as bicycle races, golf tournaments and basketball championships, are the most popular forms of races. Races studied in economic theory include patent races and contests for job promotion. Despite its importance, the theoretical literature on dynamic competition has been relatively sparse. Harris and Vickers (1987) is a pioneering paper with a model in discrete state-space. In their model, they prove that at least one equilibrium exists and characterize some of its properties. However, they only prove the uniqueness of the symmetric equilibria and they do not allow for discounting. This paper introduces a continuous time, continuous state-space model based on Harris and Vickers (1987)'s tug-of-war to address the question of the existence and uniqueness of equilibrium. The existence and uniqueness theorems apply for some cases with asymmetric players and discounting.

In this model, two players compete for a final reward. The reward is won by the first player who achieves a given distance over his rival. At any moment when the race is going on, each player puts in effort which influences the distance between him and his rival: a Brownian motion with a drift that depends on the efforts of the players. The cost of effort functions are strictly convex. I consider the set of Markovian Perfect Equilibria (MPEs) in which equilibrium strategies of the players are conditioned only on the current distance between them. I prove the existence and uniqueness of equilibrium MPE strategies under some weak conditions on the cost functions and final rewards. In some special cases, the continuous time framework delivers a closed-form solution of MPEs which facilitates the characterization of equilibrium strategies ${ }^{1}$.

The equilibrium strategies share basic properties with the equilibrium strategies in the discrete state space model in Harris and Vickers (1987). As in the latter, the Markov Perfect Equilibrium (MPE) strategies exhibit a discouragement effect: The players exert high effort only when they are close to each other. When a player is left further behind by his rival, he reduces his effort given his slim chance of winning. The rival who gets further ahead therefore faces less competition and can safely reduce his effort. A larger distance between the two players thus discourages both players. Harris and Vickers (1987) however show the discouragement effect only for the case in which two players have the same cost function and they do not discount the future. In contrast, I use the theory of boundary value problems for systems of second-order differential equations developed in Hartman (1964); this theory allows me to consider the model an show the discouragement effect in full generality with potentially asymmetric players and with discounting.

Moscarini and Smith (2007) is the first paper to address the optimal design of the race in a similar continuous time, continuous state-space model. Moscarini and Smith take a different approach to solving the model, relying on the symmetry of Markov Perfect Equilibria. Besides restricting their attention to symmetric equilibria, the authors consider only the case of no discounting.

Budd, Harris, and Vickers (1993) also solve a similar model using boundary value representations. Their method only applies when the discount rate $r$ goes to infinity. Another

\footnotetext{
${ }^{1}$ In the case with identical players, Moscarini and Smith (2007) find a closed-form identical to mine up to an affine transformation. Their method relies on symmetric equilibria. My method covers asymmetric equilibria also.
} 
continuous time continuous state-space version of the Harris and Vickers model is developed in Horner (1999). He restricts the action space to be finite, allowing for only two levels of effort. Hence, the MPE strategies are such that players switch their actions only infrequently based on some threshold rule.

In the next section, I present the model. In Section 3, I prove the existence and uniqueness of MPEs with general cost functions under some weak restrictions, both with and without discounting. In Section 4, I study the properties of equilibrium strategies including the discouragement effect. I also illustrate these properties for the case of quadratic cost functions. Section 5 concludes.

\section{The Model}

Two players, $A$ and $B$, engage in a contest for a final reward in continuous time. At each moment, each player chooses an effort, $x_{A}$ for player $A$ which $\operatorname{costs}$ him $c_{A}\left(x_{A}\right)$ and $x_{B}$ for player $B$ which costs him $c_{B}\left(x_{B}\right)$. In Harris and Vickers (1987), $A$ and $B$ are two research firms competing for an exclusive patent. The effort can then be interpreted as money spent on laboratories, equipment, researchers, etc. The players discount future costs and rewards at (potentially different) rates $r_{i} \geq 0$.

Let $z \in \mathbf{R}$ denote the relative distance between the two players. The race starts at $z=0$, and a player wins the race if he attains a certain lead over the other player: Player $A$ wins the race with reward $P_{A}$ when he reaches his lead over $B, z=K_{A}>0$, and $B$ wins the race with reward $P_{B}$ when he reaches his lead $\left(-K_{B}\right)$ over $A$, i.e., $z=K_{B}<0$. Therefore, $z$ is also the only payoff relevant state of the race. ${ }^{2}$

The uncertainty is incorporated in the temporal evolution of the state $z_{t}$ :

$$
d z_{t}=\left(x_{A t}-x_{B t}\right) d t+\sigma d W_{t}
$$

where $W_{t}$ is a standard Brownian motion and $W_{0}=0$.

In order to ensure that in each moment the effort choice of each player is a well-defined maximization problem with a unique solution, I impose the following standard assumption on the cost function of each player

Assumption 1 The cost functions $c_{i}(x)$ are twice continuously differentiable, strictly increasing and strictly convex: $c_{i}^{\prime}(x)>0$ and $c_{i}^{\prime \prime}(x)>0 \forall x>0$ and $i \in\{A, B\}$. Moreover the players do not bear any cost if they do not exert effort, $c_{i}(0)=0$ and the Inada conditions

\footnotetext{
${ }^{2}$ In this model, the only payoff relevant state is the distance between the two players because the outcome of the race only depends on the distance. While this model might be a suitable description of some type of races such a race for job promotion or tie-breaks in tennis, it is not a good model for patent races in which a player wins if he achieves a certain discovery, not his progress relative to the other player. In Cao (2009), I develop a model for this situation. The payoff relevant state is a vector of two numbers, distance of each player to a finish line. However, that model is less tractable, and I can only solve it numerically. The equilibrium strategies in this race exhibits similar properties to the equilibrium strategies in this paper. Moreover, in discrete state spaces, Harris and Vickers (1987) conjecture and verify numerically that the tug-of-war race is a close approximation of the two dimension race. Similar conjecture holds for continuous state-space.
} 
at 0 and $\infty$ are satisfied:

$$
c_{i}^{\prime}(0)=0 \text { and } \lim _{x \longrightarrow+\infty} c_{i}^{\prime}(x)=+\infty
$$

In addition, in order for the stochastic differential equation (1) describing the evolution of the state variable $z$ to have a solution, the effort choices $x_{i, t}$ must be bounded. I will impose an explicit bound on the effort choice of each player: $0 \leq x_{i, t} \leq \bar{x}$. The following assumption guarantees that $\bar{x}$ can be chosen large enough so that it is never binding in equilibrium.

Assumption $2 \int^{+\infty} \frac{d s}{\left(c_{A}^{\prime}\right)^{-1}(s)+\left(c_{B}^{\prime}\right)^{-1}(s)}=+\infty$

This assumption means that the marginal cost increases fast enough at high levels of effort so that the players never want to exert too much effort. It will be shown later in the appendix that $\bar{x}$ can be chosen as $\max \left\{\left(c_{A}^{\prime}\right)^{-1}(M),\left(c_{B}^{\prime}\right)^{-1}(M)\right\}$ in which $M>\frac{P_{A}+P_{B}}{K_{A}-K_{B}}$ is uniquely determined as

$$
\int_{\frac{P_{A}+P_{B}}{K_{A}-K_{B}}}^{M} \frac{s d s}{\frac{2 \max \left\{r_{A} P_{A}, r_{B} P_{B}\right\}}{\sigma^{2}}+\frac{2}{\sigma^{2}}\left(\left(c_{A}^{\prime}\right)^{-1}(s)+\left(c_{B}^{\prime}\right)^{-1}(s)\right) s}=P_{A}+P_{B} .
$$

Such an $M$ exists due to Assumption 2. ${ }^{3}$ This assumption is satisfied if $c_{i}^{\prime \prime}(x)$ are bounded below from 0 at infinity; i.e., there exists an $\delta$ and an $x^{*}>0$ such that $c_{i}^{\prime \prime}(x)>\delta \forall x>x^{*}$. Geometric cost functions $c_{i}(x)=c_{i} x^{k_{i}}$ with $k_{i} \geq 2$ satisfy this assumption, in particular quadratic cost functions satisfy this assumption since they have constant second derivatives.

If this assumption is not satisfied, for example, in the extreme, when they are both linear, players will exert high effort and might reach any upper bound on the efforts. I rule out this situation to avoid imposing any ad-hoc bound on effort of the players. Horner (1999) is an example of races in which $c_{i}($.$) are linear. In equilibrium, the players only choose between$ two levels of effort which can be interpreted as the bounds that he imposes on the efforts of the players given the linearity of the cost functions. The equilibrium strategies are such that players switch their actions only infrequently based on some threshold rule. This structure of equilibria is thus very different from equilibria in my model.

Under the restrictions on the cost functions and the effort choice of the players, the expected payoff functions of each player $i$ following a optimal strategy $X_{i}=\left\{x_{i t}\right\}_{t=0}^{\infty}$ given his rival's strategy $X_{-i}=\left\{x_{-i t}\right\}_{t=0}^{\infty}$ is well-defined:

$$
J_{i}(z)=\sup _{X_{i}} \mathbf{E}_{0}\left[e^{-r_{i} \tau} P_{i} \mathbf{1}_{\{i \text { wins }\}}-\int_{0}^{\tau} e^{-r t} c_{i}\left(x_{i t}\right) d t \mid z_{0}=z, X_{-i}\right],
$$

where $\tau$ is the finish time of the race, i.e., the first time where either $z_{t}$ reaches $K_{A}$, player $A$ wins the race, or $z_{t}$ reaches $K_{B}$, player $B$ wins the race. The indicator function indicates who wins the race. Notice that $\tau$ is a random variable depending on the uncertain evolution

\footnotetext{
${ }^{3}$ Harris and Vickers $(1987$, p7) also assumes this. However, they do not show conditions under which the bound is not binding.
} 
of the race; or more precisely, it is a stopping time. The race starts at $z_{0} \in\left(K_{B}, K_{A}\right)$. There are two components of the payoff functions. The first part is the discounted reward $e^{-r \tau} P_{i}$ if player $i$ wins the race, and the second part is the discounted cost of effort, $e^{-r t} c_{i}\left(x_{i t}\right)$, that player $i$ continuously makes during the race. Each player chooses a strategy maximizing his expected payoff given his rival's strategy.

The problem now is to find the equilibrium strategy functions, $\left(X_{A}, X_{B}\right)$ such that each player maximizes his expected payoff given his rival's strategy. It is well-known that the best response to a Markov strategy is a Markov strategy thus any MPE is a subgame perfect equilibrium. An analogy for continuous time games is that, if strategy $x_{B t}$ is Markovian, i.e. function of $z_{t}$ only, then $x_{A t}$ can be chosen from the class of Markovian strategies, and vice versa; therefore, I can restrict myself to cases where both strategies are Markovian.

I further restrict myself to the set of equilibria with twice differentiable value functions in order to write the second derivatives. We can then obtain the Hamilton-Jacobi-Bellman equations using the dynamic programming principle:

$$
\max _{0 \leq x_{i}\left(z_{t}\right) \leq \bar{x}}\left(-c_{i}\left(x_{i}\left(z_{t}\right)\right)-r_{i} J_{i}\left(z_{t}\right)+\left(x_{A}\left(z_{t}\right)-x_{B}\left(z_{t}\right)\right) J_{i}^{\prime}\left(z_{t}\right)+\frac{\sigma^{2}}{2} J_{i}^{\prime \prime}\left(z_{t}\right)\right)=0 .
$$

At each moment, the effort choice of each player involves the trade-off between the current convex cost of effort, $-c_{i}(x)$ with higher chance of winning, taken the other player's strategy as given, $\left(x-x_{-i}\left(z_{t}\right)\right) J_{i}^{\prime}\left(z_{t}\right)$. Each player also discounts the future payoff, $-r J_{i}\left(z_{t}\right)$, and takes into account the uncertainty evolution of the state $z, \frac{\sigma^{2}}{2} J_{i}^{\prime \prime}\left(z_{t}\right)$. The first order conditions from (4) determine the effort levels of players as functions of the derivatives of their value functions:

$$
\left(x_{A}(z), x_{B}(z)\right)=\left(f_{A}\left(J_{A}^{\prime}(z)\right), f_{B}\left(-J_{B}^{\prime}(z)\right)\right)
$$

where

$$
f_{i}(k)= \begin{cases}0 & \text { if } k \leq 0 \\ \min \left\{\left(c_{i}^{\prime}\right)^{-1}(k), \bar{x}\right\} & \text { otherwise }\end{cases}
$$

Finally, the boundary conditions for $J_{A}$ and $J_{B}$ are

$$
J_{i}\left(K_{i}\right)=P_{i}, J_{-i}\left(K_{i}\right)=0 .
$$

These boundary conditions are intuitive: when $A$ is $K_{A}$ ahead of $B$, he wins the reward $P_{A}, B$ receives nothing and when $B$ is $-K_{B}$ ahead of $A$, he wins the reward $P_{B}, A$ receives nothing.

Definition 1 A Markov Perfect Equilibrium (MPE) is a pair of equilibrium payoff functions $\left(J_{A}(z), J_{B}(z)\right)$ satisfying the Hamilton-Jacobi-Bellman equations (4) and the boundary conditions (7) and a pair of equilibrium strategies $\left(x_{A}(z), x_{B}(z)\right)$ given by (5).

The task of finding MPE strategies becomes solving a second-order boundary value problem on $\left(J_{A}(z), J_{B}(z)\right)$. We first solve the effort choice given the incentive $J_{i}^{\prime}(z)$ as in (5).Then, by plugging the effort choice into (4), we can re-write the Hamilton-JacobiBellman equations as an explicit second-order boundary value problem:

$$
\left(\begin{array}{c}
J_{A}^{\prime \prime} \\
J_{B}^{\prime \prime}
\end{array}\right)=\frac{2}{\sigma^{2}}\left(\begin{array}{c}
r_{A} J_{A} \\
r_{B} J_{B}
\end{array}\right)+\frac{2}{\sigma^{2}} F\left(\begin{array}{c}
J_{A}^{\prime} \\
J_{B}^{\prime}
\end{array}\right)
$$


with the boundary conditions (7) and where

$$
F\left(\begin{array}{c}
J_{A}^{\prime} \\
J_{B}^{\prime}
\end{array}\right)=\left(\begin{array}{c}
\left(f_{A}\left(J_{A}^{\prime}\right)-f_{B}\left(-J_{B}^{\prime}\right)\right) J_{A}^{\prime}-c_{A}\left(f_{A}\left(J_{A}^{\prime}\right)\right) \\
\left(f_{A}\left(J_{A}^{\prime}\right)-f_{B}\left(-J_{B}^{\prime}\right)\right) J_{B}^{\prime}-c_{B}\left(f_{B}\left(-J_{B}^{\prime}\right)\right)
\end{array}\right)
$$

Rewriting the Hamilton-Jacobi-Bellman equations as a boundary value problem allows me to use the theory of boundary value for systems of second order differential equation developed in Hartman (1964). Using this system, some preliminary properties of the payoff functions can be shown. First, the payoff functions are strictly positive except at the two boundaries. This is because, whenever the race is not yet concluded, a player can choose to stay in the race and to exert no effort, but he still has a positive probability of winning due to the uncertain evolution of the state $z$. Second, the closer a player is to his goal, the higher his expected payoff is because he has more chance of winning. Hence, the slope of the payoff function, which is the incentive determining the effort level of each player, is strictly positive in absolute value. So (6) implies that each player will exert a strictly positive effort at any moment of the race, that is, $x_{i}(z)>0 \forall z$ and $i \in\{A, B\}$.

Lemma 1 A solution of the payoff functions $\left(J_{A}, J_{B}\right)$ to the system (8) satisfies

1. Strict positivity of the payoff functions: $J_{A}(z), J_{B}(z)>0$ for all $z \in\left(K_{B}, K_{A}\right)$. Given the option to exert no effort, and the Brownian evolution of the distance between the two players, each player has a strictly positive probability of winning the race without incurring any cost of effort; their payoff functions are thus strictly positive whenever the race is not yet concluded.

2. Strict positivity of incentives: $J_{A}^{\prime}(z)>0$ and $J_{B}^{\prime}(z)<0$ for all $z \in\left(K_{B}, K_{A}\right)$. As each player moves closer to his goal, he has a higher probability of winning the race, therefore, his payoff is higher. Since the incentives are strictly positive, the players always exert a strictly positive level of effort.

Proof. In the Appendix, using the Gronwall's Inequality.

\section{Existence and Uniqueness of Markov Perfect Equi- librium}

Before analyzing the equilibrium strategies and outcomes of the race, it is important to prove the existence of Markov Perfect Equilibria and their uniqueness, or equivalently the existence and uniqueness of the solution to the boundary value problem (8). The steps of the existence and uniqueness proof are in the Appendix.

Theorem 1 A Markov equilibrium exists.

Proof. In the Appendix. It is enough to show that the boundary value problem (8) has at least one solution

As in other economic models, it is more difficult to ensure the uniqueness of equilibria. As a result, an extra condition, in addition to Assumption 2 on the cost of effort functions, is required to ensure the uniqueness of the MPE. 
Theorem 2 Suppose that $c_{i}^{\prime \prime}(x)$ are bounded below, i.e., there exists an $\delta$ such that

$$
c_{i}^{\prime \prime}(x)>\delta \forall x
$$

Then (8) has a unique solution when

$$
\max \left\{\left(c_{A}^{\prime}\right)^{-1}(M),\left(c_{B}^{\prime}\right)^{-1}(M)\right\}+\frac{M}{\delta}<4 \min \left\{r_{A}, r_{B}\right\}+\sigma^{2} \frac{\pi^{2}}{\left(K_{A}-K_{B}\right)^{2}},
$$

where $M$ is determined in (2).

Proof. In the Appendix.

Notice that the left hand side of (10) is strictly increasing in $M$ and the (2) implies that $M$ is increasing in $\max \left(P_{A}, P_{B}\right)$, so the solution is unique if $\max \left(P_{A}, P_{B}\right)$ is relatively small, the discount factor is relatively high or the degree of uncertainty, $\sigma$, is relatively large. Finally, as in Harris and Vickers (1987), the race is more likely to admit a unique equilibrium if the cost function is sufficiently convex, i.e., $\delta$ is sufficiently large.

\section{The Discouragement Effect}

The previous section establishes the general existence and uniqueness of the MPE. In this section, I investigate some properties of the MPE strategies. A striking property is that higher distance between the leader and the follower discourages both from exerting effort, which is often mentioned as the discouragement effect. This effect leads to an ambiguous effect of incentives, such as the effect of higher final reward to the winner of the race on the total expected effort of the players. Moscarini and Smith (2007) show that a higher final reward does not necessarily increase the total expected effort of the players. In Cao (2009), I show that also due to this discouragement effect, handicapping the advantaged player might counter-intuitively reduce the expected completion time of the race.

In Subsection 4.1, I show the discouragement effect for general cost functions. I illustrate this effect with a special case of quadratic cost functions in Subsection 4.2 where I can find a closed form solution. The factors that affect the intensity of this effect are the final rewards, the amount of uncertainty, the level of the cost of effort to the two players.

\subsection{General cost functions}

I find two properties of the MPE strategies which are similar to the discrete time MPE strategies in Harris and Vickers (1987). First, the leader in the race puts in higher efforts than the follower does. Second, efforts of both players decrease as the gap between them increases. Other R\&D competition models share the second property of MPE strategies. For instance, Aghion, Harris, Howitt, and Vickers (2001) and Acemoglu and Akcigit (2008), both find that effort is highest when firms are technologically close to each other. The first property, however, does not hold in all models. For instance, the models of Acemoglu and Akcigit (2008) and Reinganum (1983) have the opposite property. In their model, there is an Arrow's replacement effect, i.e., the leading firm receives flow profits before successful new 
innovations, so it has relatively weaker incentive than the follower to stochastically shorten the random time to the next innovation. In contrast, in my model, players only receive reward at the end; the Arrow's replacement effect is thus not present.

To formalize the discouragement effect, I define the pivot of the game, $z^{*}$, as where the two players exert the same effort level, i.e. $x_{A}\left(z^{*}\right)=x_{B}\left(z^{*}\right)$. If there does not exist such a $z^{*}$ then either, $x_{A}(z)>x_{B}(z)$ for all $z \in\left[K_{B}, K_{A}\right]$, we define $z^{*}=K_{B}$, or $x_{A}(z)<x_{B}(z)$ for all $z \in\left[K_{B}, K_{A}\right]$, we define $z^{*}=K_{A}$. So $z^{*}$ always exists. In a symmetric equilibrium, as in Moscarini and Smith (2007), $z^{*}=0$. Harris and Vickers (1987) only prove the discouragement effect for the case in which $c_{A} \equiv c_{B}$. In such a case, $z^{*}$ can be defined equivalently as $z^{*}=\underset{z \in\left[K_{B}, K_{A}\right]}{\arg \min }\left(J_{A}(z)+J_{B}(z)\right)$.

The following lemma shows that $z^{*}$ is unique.

Lemma $2 x_{A}(z)>x_{B}(z)$ for all $z \in\left(z^{*}, K_{A}\right]$ and $x_{A}(z)<x_{B}(z)$ for all $z \in\left[K_{B}, z^{*}\right)$.

\section{Proof. Appendix}

Given this pivot $z^{*}$, we can say that, at a moment $t, A$ is the leader of the race if he is relatively closer to his goal than $B$ (the follower) is, that is, $z_{t}>z^{*}$ and vice versa when $z_{t}<z^{*}$. Given the potential asymmetries between the two players, $z^{*}$ does not necessarily correspond to the point $\frac{K_{A}+K_{B}}{2}$ where the two players are at equal absolute distances from their goal, except for symmetric equilibria.

Lemma 2 then means that the leader always exerts higher effort than the follower does. Also using the pivot, we can state the discouragement effect in the following proposition.

Proposition 1 Suppose that $z>z^{*}$, that is, player $A$ is the leader and player $B$ is the follower, then

1. The discouragement effect on the follower: The follower, player B, reduces his effort as he gets further behind. That is, $x_{B}(z)$ is strictly decreasing in $z$.

2. The discouragement effect on the leader: Suppose $r_{A}=0$, once the leader, player $A$, starts slowing down at $z$, he will continue to do so at any $\widetilde{z}>z$. That is if $x_{A}^{\prime}(z) \leq 0$ then $x_{A}^{\prime}(\widetilde{z})<0$ for all $\widetilde{z}>z^{4}$

\section{Proof. Appendix}

Let us take a closer look at the interaction between the players' impatience and strategic motives in their effort choice. When a player is behind, the discouragement effect and discounting both serve to lower effort provision (part 1 of Proposition 1). However, when a player is sufficiently ahead, the strategic motivation reduces his incentive to provide greater effort, whereas discounting operates in the opposite direction. Indeed, numerical analysis shows that when the leader's discount rate is sufficiently high, the impatience is strong enough to cancel the slowing down interval in which the leader reduces his effort after getting further ahead from the follower. Consequently, part 2 of Proposition 1 requires the additional assumption that $r_{A}=0$.

\footnotetext{
${ }^{4}$ We can also show that, if $z^{*}$ is strictly interior, intially player $A$ will increase his effort, that is, $x_{A}^{\prime}(z)>0$ for $z$ close to $z^{*}$.
} 


\subsection{Example: Quadratic cost functions}

Consider the case of quadratic cost and no discounting. I can solve the game in closed form. Moscarini and Smith (2007) obtain a closed-form identical to the one presented here for symmetric equilibria, up to an affiine transformation. Their method relies on the symmetry of equilibria. My method to derived closed-form solutions covers asymmetric equilibria also. ${ }^{5}$ I also establish the equivalence between lower cost of effort, lower uncertainty and higher final reward.

Let the cost of effort functions be quadratic $c_{i}(x)=\frac{x^{2}}{2 \alpha_{i}}$. The higher $\alpha_{i}$ is, the less effort costs to player $i$.

Lemma 3 The Markov equilibrium strategies $\left(x_{A}(z), x_{B}(z)\right)$ have the form $\left(\sigma \widetilde{x}_{A}\left(\frac{z}{\sigma}\right), \sigma \widetilde{x}_{B}\left(\frac{z}{\sigma}\right)\right)$ where $\left(\widetilde{x}_{A}(\widetilde{z}), \widetilde{x}_{B}(\widetilde{z})\right)$ is a Markov equilibrium of the game with parameters $\widetilde{\alpha}_{A}=\widetilde{\alpha}_{B}=\widetilde{\sigma}=1$ and the rewards $\widetilde{P}_{i}=\frac{\alpha_{i} P_{i}}{\sigma^{2}}$.

Proof. Appendix

This lemma says that, holding everything else constant, a player would be indifferent between seeing its cost decreases from $\frac{x^{2}}{\alpha}$ to $\frac{x^{2}}{\alpha^{\prime}}$ and seeing the final reward augmented by $\frac{\alpha^{\prime}}{\alpha}$. Moreover, both players would be indifferent seeing the degree of uncertainty decreases from $\sigma$ to $\sigma^{\prime}$ and seeing their final rewards augmented by $\frac{\sigma}{\sigma^{\prime}}$.

The pair of the strategy functions is a solution to a vector-valued first-order boundary problem. The closed-form solution is derived in the Appendix in which show that we can implicitly solve for the effort ratio of the effort choices of the two players, $g=\frac{x_{A}(z)}{x_{B}(z)}$, as a function of the relative distance between them. In particular, $g(z)$ is the solution to the following equation:

$$
g-\frac{1}{g}+2 \ln (g)=C_{1} z+C_{2}
$$

Since $f(g)=g-\frac{1}{g}+2 \ln (g)$ is strictly increasing over the interval $(0,+\infty)$ and

$$
\lim _{g \longrightarrow 0} f(g)=-\infty, \lim _{g \longrightarrow \infty} f(g)=+\infty,
$$

for each $z$ there exists a unique $g(z)$ satisfies (11). We have $C_{1}$ greater than 0 , thus $g(z)$ is increasing in $z$, i.e., a player exerts relatively higher effort than his rival does when the former is closer to his goal. The "pivot" of the race then corresponds to $z^{*}$ where $g\left(z^{*}\right)=1$. It follow immediately from the fact that $g(z)$ is strictly increasing that the leader always exert higher effort than the followers does. The discouragement effects in Proposition 1, part1 and 2 also follow from the closed form solution of the strategy functions

$$
x_{A}=\frac{C_{1} g^{2}}{(g+1)^{3}}, x_{B}=\frac{C_{1} g}{(g+1)^{3}} .
$$

Let $z>z^{*}$. For the follower, given $g(z)$ is increasing in $z$ and $x_{B}$ is strictly increasing in $g$ if $g>\frac{1}{2}, x_{B}$ is strictly decreasing. For the leader, $x_{A}$ starts decreasing his effort at $z^{* *}$ such

\footnotetext{
${ }^{5}$ Similar derivation methods applies for geometric cost functions $c_{i}(x)=\frac{1}{\alpha_{i}} \frac{x^{1+k}}{1+k}, k>0$, however the final expressions are not as simple as the ones for quadratic cost functions.
} 
that $g\left(z^{* *}\right)=2$. So depending on whether $g\left(K_{A}\right)>2$, i.e. on $P_{i}, \alpha_{i}, \sigma$ from expression $(27)$

in the Appendix, the leader will start slowing down before the end of the race.

The higher the final reward, the stronger the discouragement effect. When $P_{A}$ and $P_{B}$ are large, the two players will both exert high effort only when they are close to each other, however when one player gets further ahead of his rival, he wants to reduce his effort because the cost of effort is too high to him. He can safely reduce his effort since the continuous time, continuous state-space and perfect information features of the race allow him to commit to engage in a war phase with high effort when his rival gets closer to him. Given this strategy, his rival also reduces effort because of the smaller chance to win the race. As both $P_{A}, P_{B}$ go to infinity, both players only exert infinitely high effort over a infinitely small distance to each other. As one of them takes the lead, the other reduces his effort to almost 0 , and the leader exerts an infinitesimal effort level as well.

Moreover, the equivalence result above shows that lower cost of effort delivers the same equilibrium strategies as if the cost of effort remains unchanged but the final rewards are higher. Thus, the lower the cost of effort to the players, the stronger the discouragement effect. Lower cost of effort allows the players to sustain their strategy more cheaply.

Finally, also by the equivalence result, a lower uncertainty on the evolution of the state of the race, i.e. lower $\sigma$, corresponds to higher $P_{A}$ and $P_{B}$, and thus a stronger discouragement effect. Indeed, the equivalent strategies are the same as in the case of unit uncertainty $\sigma=1$ and the final rewards are respectively $\widetilde{P}_{A}=\frac{P_{A}}{\sigma^{2}}$ and $\widetilde{P}_{B}=\frac{P_{B}}{\sigma^{2}}$. In the limiting case when, there is no uncertainty, i.e., $\sigma=0$, the disadvantaged player knows that the advantaged player will rationally outdo any effort he makes. This credible threat discourages the weaker player from making any effort. Fudenberg et al. (1983) and Harris and Vickers (1985) stress the same point.

\section{Conclusion}

In this paper, I develop a simple continuous time model of racing under uncertainty. I prove the existence of Markov Perfect Equilibria and, in some cases, also their uniqueness. The equilibria have similar properties to those in the original discrete time model. In addition, for some special cases, I can derive the closed-form of these MPE strategies, which facilitates the study of the comparative statics. In Cao (2009), I use this closed-form solution to show that handicapping the advantaged player in a race might be useful. A future research direction is to develop a model with more realistic features of certain races, for example, allowing for more general cost functions, discounting, and for a finish line instead of distance between players. Even though these models do not have closed-form MPEs, in Cao (2009) I show that it is still possible to numerically compute the equilibria, and examine their properties. Interestingly, the properties of the MPEs in these models are consistent with the results from the less general model. 


\section{Appendix}

Derivation of Hamilton-Jacobi-Bellman equations. For example, for firm $A$ at time $t$, assume that it optimizes from $t+\Delta t$ forward and solves

$$
\begin{aligned}
J_{A}\left(z_{t}\right) & =\max _{x} E_{t}\left[-\int_{0}^{\Delta t} e^{-r_{A} s} c_{A}\left(x_{t+s}\right) d s+e^{-r_{A} \Delta t} J_{A}\left(z_{t+\Delta t}\right)\right] \\
& =\max _{x} E_{t}\left(-\Delta t c_{A}(x)+e^{-r_{A} \Delta t}\left(J_{A}\left(z_{t}\right)+\Delta J_{A}\left(z_{t}\right)\right)+o(\Delta t)\right)
\end{aligned}
$$

The first part of the last expression is the flow of the cost of R\&D effort during a time interval of length $\Delta t$. The second part is the discounted continuation value after this time interval. The continuation value is discounted by the factor $e^{-r_{A} \Delta t}=1-r_{A} \Delta t+o(\Delta t)$, where, from now on, $o(\Delta t)$ denotes second-order terms. This continuation value depends on the evolution of $z_{t}$ to $z_{t+\Delta t}$. By Ito's Lemma, we have:

$$
\begin{aligned}
\Delta J_{A}\left(z_{t}\right)= & J_{A}\left(z_{t+\Delta t}\right)-J_{A}\left(z_{t}\right) \\
= & J_{A}^{\prime}\left(z_{t}\right) \Delta z_{t}+\frac{\sigma^{2}}{2} J_{A}^{\prime \prime}\left(z_{t}\right) \Delta t+o(\Delta t) \\
= & J_{A}^{\prime}\left(z_{t}\right)\left(x_{A t}-x_{B t}\right) \Delta t+J_{A}^{\prime}\left(z_{t}\right) \sigma \Delta W_{t} \\
& +\frac{\sigma^{2}}{2} J_{A}^{\prime \prime}\left(z_{t}\right) \Delta t+o(\Delta t) .
\end{aligned}
$$

Taking the expectations of both sides, and using the normal independent increments property of the Brownian noise, we have $E_{t}\left[J_{A}^{\prime}\left(z_{t}\right) \sigma \Delta W_{t}\right]=0$. Thus

$$
\begin{aligned}
E_{t}\left[\Delta J_{A}\left(z_{t}\right)\right]= & J_{A}^{\prime}\left(z_{t}\right)\left(x_{A t}-x_{B t}\right) \Delta t \\
& +\frac{\sigma^{2}}{2} J_{A}^{\prime \prime}\left(z_{t}\right) \Delta t+o(\Delta t) .
\end{aligned}
$$

Now, substitute these results into (13) and subtract $J_{A}\left(z_{t}\right)$ from both sides. Dividing all terms by $\Delta t$, and taking the limit as $\Delta t \longrightarrow 0$, we obtain the Hamilton-Jacobi-Bellman equation (4) for the value function of firm $A$. We obtain similarly the Hamilton-JacobiBellman equation (4) for the value function of firm $B$.

To prove Lemma 1, we will make use of the Gronwall's Inequality ${ }^{6}$ in (Hartman 1964, pg. 24).

Proof of Lema 1. 1) Let $z^{*}$ be a minimum of $J_{A}(z)$ over the interval $\left[K_{B}, K_{A}\right]$ (since $J_{A}($. is continuous, that minimum exists). If $z^{*}$ is an interior point then we have $J_{A}^{\prime}\left(z^{*}\right)=0$. From

\footnotetext{
${ }^{6}$ Grownwall's Inequality, Hartman (1964) II-1.1 Let $u(t)$ and $v(t)$ be non-negative, continuous functions on $[a, b] ; C \geq 0$ a constant; and

$$
v(t) \leq C+\int_{a}^{t} v(s) u(s) d s \text { for } a \leq t \leq b .
$$

Then

$$
v(t) \leq C \exp \left(\int_{a}^{t} u(s) d s\right) \text { for } a \leq t \leq b,
$$

in particular, if $C=0$, then $v \equiv 0$.
} 
(4) we have $J_{A}^{\prime \prime}\left(z^{*}\right)=2 r_{A} J_{A}\left(z^{*}\right)$. In addition since $z^{*}$ is a minimum, we have $J_{A}^{\prime \prime}\left(z^{*}\right) \geq 0$ so $J_{A}\left(z^{*}\right) \geq 0$. Furthermore, at the two boundaries, $J_{A} \geq 0$ therefore $J_{A}(z) \geq 0$ for all $z \in$ $\left[K_{B}, K_{A}\right]$.

Now if there exists an interior point $z^{*}$ such that $J_{A}\left(z^{*}\right)=0$, let $z^{* *}$ be a maximum of $J_{A}($.$) over \left[K_{B}, z^{*}\right]$; then $J_{A}^{\prime}\left(z^{*}\right)=0$ and $J_{A}^{\prime \prime}\left(z^{* *}\right)=2 r_{A} J_{A}\left(z^{* *}\right)$. Since $z^{* *}$ is a maximum and $J_{A}^{\prime \prime}\left(z^{* *}\right) \leq 0$, we have $J_{A}\left(z^{* *}\right) \leq 0$, thus $J_{A}(z)=0$ for all $z \in\left[K_{B}, z^{*}\right]$. And from the fact that $z^{*}$ is strictly interior,

$$
J_{A}\left(z^{*}\right)=J_{A}^{\prime}\left(z^{*}\right)=0 .
$$

We can show that this yields a contradiction because $J_{A}$ would be identically 0 over $\left[z^{*}, K_{A}\right]$. First of all, we have the following inequality:

$$
\begin{aligned}
\left|J_{A}^{\prime \prime}(z)\right| & =\frac{2}{\sigma^{2}}\left|r_{A} J_{A}(z)+c_{A}\left(f_{A}\left(J_{A}^{\prime}(z)\right)\right)-\left(f_{A}\left(J_{A}^{\prime}(z)\right)-x_{B}(z)\right) J_{A}^{\prime}(z)\right| \\
& \leq \frac{2 r_{A}}{\sigma^{2}}\left|J_{A}(z)\right|+3 \bar{x}\left|J_{A}^{\prime}(z)\right|
\end{aligned}
$$

where the inequalities is obtained from the three inequalities

$$
\begin{aligned}
0 & \leq x_{B}(z) \leq \bar{x} \\
0 & \leq f_{A}\left(J_{A}^{\prime}(z)\right) \leq \bar{x} \\
0 & \leq c_{A}\left(f_{A}\left(J_{A}^{\prime}(z)\right)\right) \\
& \leq c_{A}^{\prime}\left(f_{A}\left(J_{A}^{\prime}(z)\right)\right) f_{A}\left(J_{A}^{\prime}(z)\right) \\
& \leq\left|J_{A}^{\prime}(z)\right| \bar{x} .
\end{aligned}
$$

Apply the Gronwall's inequality for $\left|J_{A}(z)\right|^{2}+\left|J_{A}^{\prime}(z)\right|^{2}$, we have $J_{A}(z)=J_{A}^{\prime}(z)=0 \forall z \in$ $\left[z^{*}, K_{A}\right]$. This yields a contradiction with the fact that $J_{A}\left(K_{A}\right)=P_{A}>0$.

So we have $J_{A}(z)>0$ for all $z \in\left(K_{B}, K_{A}\right)$. The proof for $J_{B}(z)$ is analogous.

2) By the mean value theorem, there exists a $z^{0} \in\left(K_{B}, K_{A}\right)$ such that

$$
J_{A}^{\prime}\left(z^{0}\right)=\frac{J_{A}\left(K_{A}\right)-J_{A}\left(K_{B}\right)}{K_{A}-K_{B}}=\frac{P_{A}}{K_{A}-K_{B}}>0
$$

If there exists some $z^{1} \in\left(K_{B}, K_{A}\right)$ such that $J_{A}^{\prime}\left(z^{1}\right)<0$, then, by the intermediate value theorem, there exists an interior point $z^{*}$ between $z^{0}$ and $z^{1}$ such that $J_{A}^{\prime}\left(z^{*}\right)=0$. Hence, from the first part,

$$
J_{A}^{\prime \prime}\left(z^{*}\right)=2 r_{A} J_{A}\left(z^{*}\right)>0 .
$$

Consider the interval $\left[K_{B}, z^{*}\right]$, at $z=K_{B}, J_{A}\left(K_{B}\right)=0$. The extreme $K_{B}$ cannot be a maximum of $J_{A}$ over this interval. And in a neighborhood $z=z^{*}-\varepsilon$ of $z^{*}$,

$$
J_{A}(z)=J_{A}\left(z^{*}\right)+\frac{1}{2} J_{A}^{\prime \prime}\left(z^{*}\right) \varepsilon^{2}+o\left(\varepsilon^{2}\right)>J_{A}\left(z^{*}\right),
$$

so this extreme $z^{*}$ cannot be a maximum over the interval, either. Thus, $J_{A}$ has an interior maximum in the interval. Denote this maximum $z^{* *}$. We have $J_{A}^{\prime}\left(z^{* *}\right)=0$. This yields a 
contradiction because it implies $J_{A}^{\prime \prime}\left(z^{* *}\right)>0$, or $z^{* *}$ is a local minimum.

We have established that $J_{A}^{\prime}(z)>0 \forall z \in\left(K_{B}, K_{A}\right)$. The argument for $J_{B}^{\prime}(z)<0 \forall z \in$ $\left(K_{B}, K_{A}\right)$ is analogous.

The proof for the case $r_{A}=0$ is easier. For example, if there exists $z^{*} \in\left[K_{B}, K_{A}\right]$ such that $J_{A}^{\prime}\left(z^{*}\right)=0$, then, as derived in (14)

$$
\left|J_{A}^{\prime \prime}(z)\right| \leq 3 \bar{x}\left|J_{A}^{\prime}(z)\right| .
$$

Again, by applying the Gronwall's inequality, we have $J_{A}^{\prime}(z)=0 \forall z \in\left[K_{B}, K_{A}\right]$. But we know that,

$$
J_{A}\left(K_{B}\right)=0<P_{A}=J_{A}\left(K_{A}\right)
$$

and hence we have a contradiction. It follows that $J_{A}^{\prime}(z)>0 \forall z \in\left[K_{B}, K_{A}\right]$. The argument for $J_{B}^{\prime}(z)<0 \forall z \in\left(K_{B}, K_{A}\right)$ is analogous.

Steps of the proof of Theorem 1. The steps of the existence proof are the following. I will show that there exist constants $P, M$ and a globally bounded vector-valued function $g$ satisfying

1) $\forall\left|J_{i}\right| \leq P,\left|J_{i}^{\prime}\right| \leq M, i=A, B$

$$
g\left(\left(\begin{array}{l}
J_{A} \\
J_{B}
\end{array}\right),\left(\begin{array}{c}
J_{A}^{\prime} \\
J_{B}^{\prime}
\end{array}\right)\right)=\frac{2}{\sigma^{2}}\left(\begin{array}{c}
r_{A} J_{A} \\
r_{B} J_{B}
\end{array}\right)+\frac{2}{\sigma^{2}} F\left(\begin{array}{c}
J_{A}^{\prime} \\
J_{B}^{\prime}
\end{array}\right) .
$$

However, $g$ can be different from the right hand side outside this region 2)Any solution to the boundary value problem

$$
\begin{aligned}
\left(\begin{array}{l}
J_{A}^{\prime \prime}(z) \\
J_{B}^{\prime \prime}(z)
\end{array}\right) & =g\left(\left(\begin{array}{l}
J_{A} \\
J_{B}
\end{array}\right),\left(\begin{array}{l}
J_{A}^{\prime} \\
J_{B}^{\prime}
\end{array}\right)\right) \\
J_{i}\left(K_{i}\right) & =P_{i} \text { and } J_{i}\left(K_{-i}\right)=0 \text { for } i=A, B
\end{aligned}
$$

will satisfy $\left|J_{i}(z)\right| \leq P,\left|J_{i}^{\prime}(z)\right| \leq M, i=A, B \forall z \in\left[K_{B}, K_{A}\right]$

Therefore, any solution to the boundary value problem (15) is also a solution to the original problem (8).

In order to prove the existence and the uniqueness of the solution to the boundary problem (8), we first provide a bound on the effort intensity of each firm.

Lemma 4 There exists some $M$ depending only on $P_{A}, P_{B}, K_{A}, K_{B}$ and $c($.$) such that 0<$ $J_{A}^{\prime}(z),-J_{B}^{\prime}(z)<M \forall z \in\left(K_{B}, K_{A}\right)$

Proof. Let $D(z)=J_{A}(z)-J_{B}(z)$ then $D^{\prime}(z)=J_{A}^{\prime}(z)-J_{B}^{\prime}(z)$ and $0<J_{A}^{\prime}(z),-J_{B}^{\prime}(z)<$ $D^{\prime}(z)$. Substituting the effort functions (6) into the Hamilton-Jacobi-Bellman equation (8), we have:

$$
\begin{aligned}
& J_{A}^{\prime \prime}(z)=\frac{2 r_{A}}{\sigma^{2}} J_{A}(z)+\frac{2}{\sigma^{2}}\left(\begin{array}{c}
-c_{A}\left(\left(f_{A}\left(J_{A}^{\prime}(z)\right)\right)\right)+\left(f_{A}\left(J_{A}^{\prime}(z)\right)\right) J_{A}^{\prime}(z) \\
-\left(f_{B}\left(-J_{B}^{\prime}(z)\right)\right) J_{A}^{\prime}(z)
\end{array}\right) \\
& J_{B}^{\prime \prime}(z)=\frac{2 r_{B}}{\sigma^{2}} J_{B}(z)+\frac{2}{\sigma^{2}}\left(\begin{array}{c}
-c_{B}\left(\left(f_{B}\left(-J_{B}^{\prime}(z)\right)\right)\right)-\left(f_{B}\left(-J_{B}^{\prime}(z)\right)\right) J_{B}^{\prime}(z) \\
+\left(f_{A}\left(J_{A}^{\prime}(z)\right)\right) J_{B}^{\prime}(z)
\end{array}\right) .
\end{aligned}
$$


By subtracting these two equalities, we obtain

$$
\left|D^{\prime \prime}(z)\right| \leq \frac{2 \max \left\{r_{A} P_{A}, r_{B} P_{B}\right\}}{\sigma^{2}}+\frac{2}{\sigma^{2}}\left(\left(\left(c_{A}^{\prime}\right)^{-1}\left(D^{\prime}(z)\right)\right) D^{\prime}(z)+\left(\left(c_{B}^{\prime}\right)^{-1}\left(D^{\prime}(z)\right)\right) D^{\prime}(z)\right)
$$

where $P=\max \left(P_{A}, P_{B}\right)$. This is due to the facts that

$$
\begin{aligned}
0 & \leq-c_{A}\left(\left(f_{A}\left(J_{A}^{\prime}(z)\right)\right)\right)+\left(f_{A}\left(J_{A}^{\prime}(z)\right)\right) J_{A}^{\prime}(z) \leq\left(c_{A}^{\prime}\right)^{-1}\left(J_{A}^{\prime}(z)\right) J_{A}^{\prime}(z) \\
0 & \leq\left(f_{B}\left(-J_{B}^{\prime}(z)\right)\right) J_{A}^{\prime}(z) \leq\left(c_{B}^{\prime}\right)^{-1}\left(-J_{B}^{\prime}(z)\right) J_{A}^{\prime}(z) \\
0 & \leq-c_{B}\left(\left(f_{B}\left(-J_{B}^{\prime}(z)\right)\right)\right)-\left(f_{B}\left(-J_{B}^{\prime}(z)\right)\right) J_{B}^{\prime}(z) \leq\left(c_{B}^{\prime}\right)^{-1}\left(-J_{B}^{\prime}(z)\right)\left(-J_{B}^{\prime}(z)\right) \\
0 & \leq-\left(f_{A}\left(J_{A}^{\prime}(z)\right)\right) J_{B}^{\prime}(z) \leq\left(c_{A}^{\prime}\right)^{-1}\left(J_{A}^{\prime}(z)\right)\left(-J_{B}^{\prime}(z)\right)
\end{aligned}
$$

and

$$
\begin{aligned}
\left(c_{A}^{\prime}\right)^{-1}\left(J_{A}^{\prime}(z)\right) & \leq\left(c_{A}^{\prime}\right)^{-1}\left(D^{\prime}(z)\right) \\
\left(c_{B}^{\prime}\right)^{-1}\left(-J_{B}^{\prime}(z)\right) & \leq\left(c_{B}^{\prime}\right)^{-1}\left(D^{\prime}(z)\right) .
\end{aligned}
$$

By the mean value theorem, there exists a $z^{*} \in\left(K_{B}, K_{A}\right)$ such that $D^{\prime}\left(z^{*}\right)=\frac{P_{A}+P_{B}}{K_{A}-K_{B}}$. $\forall z \in\left[K_{B}, K_{A}\right]$. It then follows that

$$
\begin{aligned}
& P_{A}+P_{B} \\
> & \left|\int_{z^{*}}^{z} D^{\prime}(t) d t\right| \\
> & \left|\int_{z^{*}}^{z} D^{\prime}(t) \frac{D^{\prime \prime}(t)}{\frac{2 \max \left\{r_{A} P_{A}, r_{B} P_{B}\right\}}{\sigma^{2}}+\frac{2}{\sigma^{2}}\left(\left(\left(c_{A}^{\prime}\right)^{-1}\left(D^{\prime}(z)\right)\right) D^{\prime}(z)+\left(\left(c_{B}^{\prime}\right)^{-1}\left(D^{\prime}(z)\right)\right) D^{\prime}(z)\right)} d t\right| \\
& D^{\prime} \underline{\underline{t}=}=s\left|\int_{\frac{P_{A}+P_{B}}{K_{A}-K_{B}}}^{D^{\prime}(z)}\right| \frac{s d s}{\frac{2 \max \left\{r_{A} P_{A}, r_{B} P_{B}\right\}}{\sigma^{2}}+\frac{2}{\sigma^{2}}\left(\left(\left(c_{A}^{\prime}\right)^{-1}(s)\right)+\left(\left(c_{B}^{\prime}\right)^{-1}(s)\right)\right) s} \mid .
\end{aligned}
$$

The last equality is a result of a change of integration variables from $t$ to $s=D^{\prime}(t)$ : $D^{\prime}(t) D^{\prime \prime}(t) d t=D^{\prime}(t) d D^{\prime}(t)$. Assumption 2 implies that

$$
\int_{\frac{P_{A}+P_{B}}{K_{A}-K_{B}}}^{\infty} \frac{s d s}{\frac{2 \max \left\{r_{A} P_{A}, r_{B} P_{B}\right\}}{\sigma^{2}}+\frac{2}{\sigma^{2}}\left(\left(\left(c_{A}^{\prime}\right)^{-1}(s)\right)+\left(\left(c_{B}^{\prime}\right)^{-1}(s)\right)\right) s}=+\infty .
$$

Thus, there exists an $M$ such that

$$
\int_{\frac{P_{A}+P_{B}}{K_{A}-K_{B}}}^{M} \frac{s d s}{\frac{2 \max \left\{r_{A} P_{A}, r_{B} P_{B}\right\}}{\sigma^{2}}+\frac{2}{\sigma^{2}}\left(\left(\left(c_{A}^{\prime}\right)^{-1}(s)\right)+\left(\left(c_{B}^{\prime}\right)^{-1}(s)\right)\right) s}=P_{A}+P_{B} .
$$

We conclude that

$$
D^{\prime}(z)<M \forall z \in\left(K_{B}, K_{A}\right)
$$

Using these bounds on $J_{A}^{\prime}(z)$ and $J_{B}^{\prime}(z)$, we now can prove the existence of a solution for any value of $r_{i}, P_{i}, K_{i} \cdot{ }^{7}$ To this end, the following classical lemma from (G.Scorza-Dragoni 1935) will be useful:

\footnotetext{
${ }^{7}$ This proof follows closely Hartman (1960)
} 
Lemma Let $g\left(t, x, x^{\prime}\right)$ be a continuous and bounded (vector-valued) function for $0 \leq t \leq$ $T$ and arbitrary $\left(x, x^{\prime}\right)$. Then, for arbitrary $x_{0}$ and $x_{T}$ the system of differential equations

$$
x^{\prime \prime}=g\left(t, x, x^{\prime}\right)
$$

has at least one solution $x=x(t)$ satisfying

$$
x(0)=x_{0}, x(T)=x_{T} .
$$

It is been pointed out by Bass (1958) that this lemma is easily derived from the Schauder's fixed point theorem. In order to use this lemma, we need to transform the system (8) into a bounded system over $\left(\begin{array}{l}J_{A} \\ J_{B}\end{array}\right)$ and $\left(\begin{array}{c}J_{A}^{\prime} \\ J_{B}^{\prime}\end{array}\right)$.

Proof of the Theorem 1. First, we can easily find two bounded, strictly increasing and infinitely differentiable functions $\varphi, \chi$ such that

$$
\begin{aligned}
& \varphi(x)=x \text { if }|x| \leq P \text { and }\left|\varphi^{\prime}\right| \leq 1 \\
& \chi(x)=x \text { if }|x| \leq M \text { and }\left|\chi^{\prime}\right| \leq 1
\end{aligned}
$$

Consider the function

$$
\begin{aligned}
& g\left(\left(\begin{array}{c}
J_{A} \\
J_{B}
\end{array}\right),\left(\begin{array}{c}
J_{A}^{\prime} \\
J_{B}^{\prime}
\end{array}\right)\right) \\
= & \frac{2}{\sigma^{2}}\left(\begin{array}{c}
r_{A} \varphi\left(J_{A}\right) \\
r_{B} \varphi\left(J_{B}\right)
\end{array}\right)+\frac{2}{\sigma^{2}} F\left(\begin{array}{c}
\chi\left(J_{A}^{\prime}\right) \\
\chi\left(J_{B}^{\prime}\right)
\end{array}\right) .
\end{aligned}
$$

Since $\varphi, \chi$ are bounded $g$ is bounded, then by the Lemma from (G.Scorza-Dragoni 1935), the boundary value problem

$$
\begin{aligned}
\left(\begin{array}{c}
J_{A}^{\prime \prime} \\
J_{B}^{\prime \prime}
\end{array}\right) & =g\left(\left(\begin{array}{c}
J_{A} \\
J_{B}
\end{array}\right),\left(\begin{array}{c}
J_{A}^{\prime} \\
J_{B}^{\prime}
\end{array}\right)\right) \\
\left(\begin{array}{c}
J_{A}\left(K_{B}\right) \\
J_{B}\left(K_{B}\right)
\end{array}\right) & =\left(\begin{array}{c}
0 \\
P_{B}
\end{array}\right),\left(\begin{array}{c}
J_{A}\left(K_{A}\right) \\
J_{B}\left(K_{A}\right)
\end{array}\right)=\left(\begin{array}{c}
P_{A} \\
0
\end{array}\right)
\end{aligned}
$$

has at least one solution $\left(\begin{array}{l}J_{A}(z) \\ J_{B}(z)\end{array}\right) K_{B} \leq z \leq K_{A}$. We can proceed exactly the same way as in the proof of Lemma 1 and 4 to show that $0<J_{A}(z), J_{B}(z)<P$ and $0<J_{A}^{\prime}(z),-J_{B}^{\prime}(z)<$ $M$, so $\left(\begin{array}{l}J_{A}(z) \\ J_{B}(z)\end{array}\right) K_{B} \leq z \leq K_{A}$ is also the solution to the original system.

In order to prove uniqueness, we use theorem XII-4.3 ${ }^{8}$ from (Hartman 1964, pg. 425).

\footnotetext{
${ }^{8}$ Theorem XII-4.3 (Hartman 1964, pg 425 )Let $f\left(t, x, x^{\prime}\right)$ be continuous for $0 \leq t \leq p$ and for $\left(x, x^{\prime}\right)$ on some $2 \mathrm{~d}$-dimensional convex set. Let $f\left(t, x, x^{\prime}\right)$ have continuous partial derivatives with respect to the components of $x$ and $x^{\prime}$. Let the Jacobian matrices of $f$ with respect to $x, x^{\prime}$

$$
\begin{aligned}
& B\left(t, x, x^{\prime}\right)=\partial_{x} f\left(t, x, x^{\prime}\right) \\
& F\left(t, x, x^{\prime}\right)=\partial_{x^{\prime}} f\left(t, x, x^{\prime}\right)
\end{aligned}
$$
}


Proof of Theorem 2. In order to apply the Theorem XII - 4.3 (Hartman 1964, pg. 425), we need to verify the condition

$$
2\left(B-\frac{1}{4} F F^{*}\right) s . s>-\frac{\pi^{2}}{\left(K_{A}-K_{B}\right)^{2}}\|s\|^{2},
$$

where

$$
B\left(J, J^{\prime}\right)=\left(\begin{array}{cc}
\frac{2 r_{A}}{\sigma^{2}} & 0 \\
0 & \frac{2 r_{B}}{\sigma^{2}}
\end{array}\right)
$$

and

$$
F\left(J, J^{\prime}\right)=\frac{2}{\sigma^{2}}\left(\begin{array}{cc}
\left(c_{A}^{\prime}\right)^{-1}\left(J_{A}^{\prime}\right)-\left(c_{B}^{\prime}\right)^{-1}\left(-J_{B}^{\prime}\right) & -\frac{J_{A}^{\prime}}{c_{B}^{\prime \prime}\left(\left(c_{B}^{\prime}\right)^{-1}\left(-J_{B}^{\prime}\right)\right)} \\
-\frac{J_{B}^{\prime}}{c_{A}^{\prime \prime}\left(\left(c_{A}^{\prime}\right)^{-1}\left(J_{A}^{\prime}\right)\right)} & \left(c_{A}^{\prime}\right)^{-1}\left(J_{A}^{\prime}\right)-\left(c_{B}^{\prime}\right)^{-1}\left(-J_{B}^{\prime}\right)
\end{array}\right) .
$$

Given that $0<J_{A}^{\prime},-J_{B}^{\prime}<M$ we have

$$
\left(F F^{*} s\right) . s \leq \frac{1}{\sigma^{2}}\left(\max \left\{\left(c_{A}^{\prime}\right)^{-1}(M),\left(c_{B}^{\prime}\right)^{-1}(M)\right\}+\frac{M}{\delta}\right)\|s\|^{2} .
$$

Together with $(B s) . s \geq \frac{2 \min \left\{r_{A}, r_{B}\right\}}{\sigma^{2}}\|s\|^{2}$, we have (10) implies (16).

Proof of Lemma 2. First we show that, whenever $x_{A}(z)=x_{B}(z)=x$, we have

$$
x_{A}^{\prime}(z)>0>x_{B}^{\prime}(z) .
$$

Indeed, we have

$$
x_{A}^{\prime}(z)=\frac{d}{d z}\left(\left(c_{A}^{\prime}\right)^{-1}\left(J_{A}^{\prime}(z)\right)\right)=\frac{J_{A}^{\prime \prime}(z)}{c_{A}^{\prime \prime}\left(x_{A}(z)\right)} .
$$

Using $J_{A}^{\prime \prime}(z)$ from (4) we have

$$
\begin{aligned}
x_{A}^{\prime}(z) & =\frac{2}{\sigma^{2}} \frac{-\left(x_{A}(z)-x_{B}(z)\right) J_{A}^{\prime}(z)+r_{A} J_{A}(z)+c_{A}\left(x_{A}(z)\right)}{c_{A}^{\prime \prime}\left(x_{A}(z)\right)} \\
& =\frac{2}{\sigma^{2}} \frac{r_{A} J_{A}(z)+c_{A}(x)}{c_{A}^{\prime \prime}(x)}>0 .
\end{aligned}
$$

satisfy

$$
2\left(B-\frac{1}{4} F F^{*}\right) \mathbf{z} \cdot \mathbf{z}>-\frac{\pi^{2}}{p^{2}}\|\mathbf{z}\|^{2}
$$

for all constant vectors $\mathbf{z} \neq \mathbf{0}$. Then the boundary value problem

$$
\begin{aligned}
x^{\prime \prime} & =f\left(t, x, x^{\prime}\right) \\
x(0) & =x_{0} \\
x(p) & =x_{p}
\end{aligned}
$$

has at most one solution. 
Similarly

$$
\begin{aligned}
x_{B}^{\prime}(z) & =\frac{d}{d z}\left(\left(c_{B}^{\prime}\right)^{-1}\left(-J_{B}^{\prime}(z)\right)\right)=\frac{-J_{B}^{\prime \prime}(z)}{c_{B}^{\prime \prime}\left(x_{B}(z)\right)} \\
& =-\frac{2}{\sigma^{2}} \frac{r_{B} J_{B}(z)+c_{B}(x)}{c_{B}^{\prime \prime}(x)}<0 .
\end{aligned}
$$

So (17) at $z=z^{*}$ implies that $x_{A}^{\prime}\left(z^{*}\right)>x_{B}\left(z^{*}\right)$. As a result, there exists an $\epsilon>0$ such that $x_{A}(z)>x_{B}(z)$ over $\left(z^{*}, z^{*}+\epsilon\right)$. We prove the lemma by contradiction. Suppose that there exists an $z>z^{*}+\epsilon$ such that $x_{A}(z) \leq x_{B}(z)$. Let $z^{* *}$ be the infimum of these $z$ 's, we have

$$
x_{A}\left(z^{* *}\right)=x_{B}\left(z^{* *}\right) .
$$

However, (17) at $z=z^{* *}$ implies that $x_{A}^{\prime}\left(z^{* *}\right)>x_{B}\left(z^{* *}\right)$, so there exists an $\epsilon^{\prime}>0$ such that $x_{A}(z)<x_{B}(z)$ over $\left(z^{* *}-\epsilon^{\prime}, z^{* *}\right)$. This contradicts the fact that $z^{* *}$ is the infimum. So $x_{A}(z)>x_{B}(z)$ for all $z>z^{*}$. Similarly we have $x_{A}(z)<x_{B}(z)$ for all $z<z^{*}$.

Proof of Proposition 1. 1) We rewrite equation (4) for $B$ :

$$
-r_{B} J_{B}(z)-c_{B}\left(x_{B}(z)\right)+\left(x_{A}(z)-x_{B}(z)\right) J_{B}^{\prime}(z)+\frac{\sigma^{2}}{2} J_{B}^{\prime \prime}(z)=0 .
$$

SO

$$
\frac{\sigma^{2}}{2} J_{B}^{\prime \prime}(z)=r_{B} J_{B}(z)+c_{B}\left(x_{B}(z)\right)+\left(x_{A}(z)-x_{B}(z)\right)\left(-J_{B}^{\prime}(z)\right)>0 .
$$

Given that $x_{A}(z)>x_{B}(z)$ and $J_{B}^{\prime}(z)<0$, together with $J_{B}(z)>0$ and $c_{B}\left(x_{B}(z)\right)>0$ from Lemma 1 , we have $J_{B}^{\prime \prime}(z)>0$. As a result, $J_{B}^{\prime}(z)$ is strictly increasing over $\left(z^{*}, K_{A}\right]$ or $x_{B}(z)=\left(c_{B}^{\prime}\right)^{-1}\left(-J_{B}^{\prime}(z)\right)$ is strictly decreasing over the same interval.

2) We rewrite equation (4) for $A$ :

$$
-r_{A} J_{A}(z)-c_{A}\left(x_{A}(z)\right)+\left(x_{A}(z)-x_{B}(z)\right) J_{A}^{\prime}(z)+\frac{\sigma^{2}}{2} J_{A}^{\prime \prime}(z)=0
$$

Differentiate with respect to $z$

$$
-r_{A} J_{A}^{\prime}(z)+\left\{-c_{A}^{\prime}\left(x_{A}(z)\right)+J_{A}^{\prime}(z)\right\} x_{A}^{\prime}(z)-x_{B}(z) J_{A}^{\prime \prime}(z)-x_{B}^{\prime}(z) J_{A}^{\prime}(z)+\frac{\sigma^{2}}{2} J_{A}^{\prime \prime \prime}(z)=0
$$

given that $-c_{A}^{\prime}\left(x_{A}(z)\right)+J_{A}^{\prime}(z)=0$. This simplifies to

$$
\frac{\sigma^{2}}{2} J_{A}^{\prime \prime \prime}(z)=-r_{A} J_{A}^{\prime}(z)+x_{B}(z) J_{A}^{\prime \prime}(z)+x_{B}^{\prime}(z) J_{A}^{\prime}(z) .
$$

Part 2) implies that $x_{B}^{\prime}(z)<0$ so if $J_{A}^{\prime \prime}(z) \leq 0$ and $r_{A}=0$, then $J_{A}^{\prime \prime \prime}(z) \leq 0$ or

$$
J_{A}^{\prime \prime}\left(z^{\prime}\right) \leq 0 \forall z^{\prime} \geq z
$$

that is $J_{A}^{\prime}(z)$ is decreasing over $\left(z, K_{A}\right]$. Notice that when $r_{A}>0, r_{A} J_{A}^{\prime}(z)>0$, the statement above does not always hold: Discounting reduces the discouragement effect on the leader. 
Proof of Lemma 3. Given that the upper bound $\bar{x}$ is not binding in equilibrium, (5) implies a linear relationship between efforts and slopes of the payoff functions:

$$
x_{A}(z)=\alpha_{A} J_{A}^{\prime}(z) \text { and } x_{B}(z)=-\alpha_{B} J_{B}^{\prime}(z) .
$$

As a result (9) simplifies to

$$
F\left(\begin{array}{l}
u \\
v
\end{array}\right)=\left(\begin{array}{l}
-\alpha u^{2}-2 \beta u v \\
-2 \alpha u v-\beta v^{2}
\end{array}\right)
$$

Let $\widetilde{J}_{i}(z)=\frac{\alpha_{i}}{\sigma^{2}} J_{i}(\sigma z)$, the boundary conditions become

$$
\widetilde{J}_{i}\left(\frac{K_{-i}}{\sigma}\right)=0, \widetilde{J}_{i}\left(\frac{K_{i}}{\sigma}\right)=\frac{\alpha_{i} P_{i}}{\sigma^{2}}=\widetilde{P}_{A}
$$

and the differential equations on $J_{i}(z), i \in\{A, B\}$ become

$$
\begin{aligned}
-2 r \widetilde{J}_{i}(z)+\left(\widetilde{J}_{i}^{\prime}(z)\right)^{2}+2 \widetilde{J}_{i}^{\prime}(z) \widetilde{J}_{-i}^{\prime}(z)+\widetilde{J}_{i}^{\prime \prime}(z) & =0 \\
\forall z & \in\left(K_{B}, K_{A}\right)
\end{aligned}
$$

We can see immediately that this system of equations and the boundary conditions are equivalent to the ones resulting from the game with

$$
\begin{aligned}
\widetilde{\alpha}_{A} & =\widetilde{\alpha}_{B}=\widetilde{\sigma}=1 \\
\widetilde{P}_{i} & =\frac{\alpha_{i} P_{i}}{\sigma^{2}} .
\end{aligned}
$$

Closed form Derivation. Substituting the functional forms of the cost functions into (5), we have

$$
x_{A}(z)=J_{A}^{\prime}(z) \text { and } x_{B}(z)=-J_{B}^{\prime}(z) .
$$

Denote $x=x_{A}$ and $y=x_{B}$. Differentiating both sides of $(20)$ gives $J_{A}^{\prime \prime}(z)=x^{\prime}(z)$ and $J_{B}^{\prime \prime}(z)=-y^{\prime}(z)$. Thus, we can rewrite (19) as a system of first-order differential equations with the unknown strategy functions $x$ and $y$ :

$$
\begin{aligned}
& \frac{1}{2} x^{2}-x y+\frac{1}{2} x^{\prime}=0 \\
& \frac{1}{2} y^{2}-x y-\frac{1}{2} y^{\prime}=0 .
\end{aligned}
$$

We derive the boundary conditions for (21) using Lebnitz's rule:

$$
P_{A}=J_{A}\left(K_{A}\right)-J_{A}\left(K_{B}\right)=\int_{K_{B}}^{K_{A}} J_{A}^{\prime}(z) d z
$$

and

$$
P_{B}=J_{B}\left(K_{B}\right)-J_{B}\left(K_{A}\right)=-\int_{K_{B}}^{K_{A}} J_{B}^{\prime}(z) d z
$$


We can rewrite these conditions using the strategies function $x$ and $y$ :

$$
\begin{aligned}
& \int_{K_{B}}^{K_{A}} x(z) d z=P_{A} \\
& \int_{K_{B}}^{K_{A}} y(z) d z=P_{B} .
\end{aligned}
$$

Let us define the function $g$ as

$$
g=\frac{x}{y}
$$

$g$ is well-defined since $y>0$. We will find an explicit relationship between $g$ and $y$. From the definition of $g$ we have:

$$
x^{\prime}(z)=g^{\prime}(y) y^{\prime}(z) y(z)+g(y) y^{\prime}(z) .
$$

SO

$$
g^{\prime}(y) y=g(y) \frac{1+g(y)}{1-2 g(y)} .
$$

Rewrite this in a differential form:

$$
\frac{d g(1-2 g)}{g(g+1)}=\frac{d y}{y}
$$

or equivalently

$$
d(\ln g-3 \ln (g+1))=d(\ln y) .
$$

This differential equation implies

$$
\frac{C_{1} g(y)}{(g(y)+1)^{3}}=y,
$$

where $C_{1}>0$ is a constant pinned down by the boundary conditions. So, combining (23) and (24) yields the strategy functions (12) in the text. Now with these expressions, we determine $g$ as a function of $z$. Differentiate the expression for $x$ from (12) with respect to $z$ implies

$$
x^{\prime}=g^{\prime} \frac{C_{1}(2(g+1)-3 g) g}{(g+1)^{4}} .
$$

Plugging this expression for $x^{\prime}$ into (21) and simplifying give

$$
\frac{d g}{d z}=\frac{C_{1} g^{2}}{(g+1)^{2}} .
$$

or equivalently

$$
d\left(-\frac{1}{g(z)}+2 \ln (g(z))+g(z)\right)=C_{1} d z
$$

So we obtain finally

$$
g(z)-\frac{1}{g(z)}+2 \ln g(z)=C_{1} z+C_{2}
$$


Again, the constant $C_{2}$ is pinned down by the boundary conditions. We come back to write equations determining $C_{1}$ and $C_{2}$ using (22). The previous closed-form yields

$$
\int_{K_{B}}^{K_{A}} x(z) d z=\int_{K_{B}}^{K_{A}} \frac{C_{1} g(z)^{2}}{(g(z)+1)^{3}} d z
$$

Using the change of variable $z=z(g)$ and the differential form (25). The integral on $x$ becomes

$$
\begin{aligned}
\int_{K_{B}}^{K_{A}} x(z) d z & =\int_{K_{B}}^{K_{A}} \frac{C_{1} g(z)^{2}}{(g(z)+1)^{3}} d z=\int_{g\left(K_{B}\right)}^{g\left(K_{A}\right)} \frac{1}{(g+1)} d g \\
& =\ln \left(\frac{1+g\left(K_{A}\right)}{1+g\left(K_{B}\right)}\right)=P_{A}
\end{aligned}
$$

Similarly

$$
\begin{aligned}
\int_{K_{B}}^{K_{A}} y(z) d z & =\int_{K_{B}}^{K_{A}} \frac{C_{1} g(z)}{(g(z)+1)^{3}} d z=\int_{g\left(K_{B}\right)}^{g\left(K_{A}\right)} \frac{1}{(g+1) g} d g \\
& =\ln \left(\frac{g\left(K_{A}\right)}{g\left(K_{B}\right)}\right)-\ln \left(\frac{1+g\left(K_{A}\right)}{1+g\left(K_{B}\right)}\right)=P_{B}
\end{aligned}
$$

These equations give a system of equations on $g\left(K_{A}\right)$ and $g\left(K_{B}\right)$

$$
\begin{aligned}
\frac{1+g\left(K_{A}\right)}{1+g\left(K_{B}\right)} & =\exp \left(P_{A}\right) \\
\frac{g\left(K_{A}\right)}{g\left(K_{B}\right)} & =\exp \left(P_{A}+P_{B}\right) .
\end{aligned}
$$

We can then solve for $g\left(K_{A}\right)$ and $g\left(K_{B}\right)$ explicitly in functions of $K_{A}, K_{B}, P_{A}, P_{B}$

$$
g\left(K_{A}\right)=\frac{\exp \left(P_{A}+P_{B}\right)-\exp \left(P_{B}\right)}{\exp \left(P_{B}\right)-1},
$$

and

$$
g\left(K_{B}\right)=\frac{\exp \left(P_{A}\right)-1}{\exp \left(P_{A}+P_{B}\right)-\exp \left(P_{A}\right)} .
$$

Together with the two equations (26) on $g$ at $z=K_{B}$ and $K_{A}, C_{1}$ and $C_{2}$ are then

$$
C_{1}=\frac{1}{K_{A}-K_{B}}\left(\begin{array}{c}
\frac{\exp \left(P_{A}+P_{B}\right)-\exp \left(P_{B}\right)}{\exp \left(P_{B}\right)-1} \\
-\frac{\exp \left(P_{B}\right)-1}{\exp \left(P_{A}+P_{B}\right)-\exp \left(P_{B}\right)} \\
-\frac{\exp \left(P_{A}\right)-1}{\exp \left(P_{A}+P_{B}\right)-\exp \left(P_{A}\right)} \\
+\frac{\exp \left(P_{A}+P_{B}\right)-\exp \left(P_{A}\right)}{\exp \left(P_{A}\right)-1} \\
+2 P_{A}+2 P_{B}
\end{array}\right),
$$


and

$$
C_{2}=\frac{1}{K_{A}-K_{B}}\left(\begin{array}{c}
K_{A}\left(\begin{array}{c}
\frac{\exp \left(P_{A}\right)-1}{\exp \left(P_{A}+P_{B}\right)-\exp \left(P_{A}\right)} \\
-\frac{\exp \left(P_{A}+P_{B}\right)-\exp \left(P_{A}\right)}{\exp \left(P_{A}\right)-1} \\
+2 \ln \left(\frac{\exp \left(P_{A}\right)-1}{\exp \left(P_{A}+P_{B}\right)-\exp \left(P_{A}\right)}\right)
\end{array}\right) \\
-K_{B}\left(\begin{array}{c}
\frac{\exp \left(P_{A}+P_{B}\right)-\exp \left(P_{B}\right)}{\exp \left(P_{B}\right)-1} \\
-\frac{\exp \left(P_{B}\right)-1}{\exp \left(P_{A}+P_{B}\right)-\exp \left(P_{B}\right)} \\
+2 \ln \left(\frac{\exp \left(P_{A}+P_{B}\right)-\exp \left(P_{B}\right)}{\exp \left(P_{B}\right)-1}\right)
\end{array}\right)
\end{array}\right) .
$$




\section{References}

Acemoglu and Akcigit (2008, February). State-dependent intellectual property rights policy. MIT Working Paper.

Aghion, P., C. Harris, P. Howitt, and J. Vickers (2001). Competition, imitation and growth with step-by-step innovation. Reviews of Economic Studies 68, 467-492.

Bass (1958). On non-linear repulsive forces. Contributions to the theory of non-linear oscillations 4, 201-211.

Budd, C., C. Harris, and J. Vickers (1993). A model of the evolution of duopoly: Does the asymmetry between firms tend to increase or decrease? Reviews of Economic Studies 60, 543-573.

Cao, D. (2009, September). Racing under uncertainty: When should we handicap the advantaged competitor? MIT Working Paper.

Fudenberg et al. (1983). Pre-emption, leapfrogging and competition in patent races. European Economic Review 22, 3-31.

G.Scorza-Dragoni (1935). Sul problema dei valori ai limiti per i systemi di equazioni differenziali del secondo ordine. Boll.Un.Mat.Ital 14, 225-230.

Harris and Vickers (1985). Perfect equilibrium in a model of a race. Reviews of Economic Studies 53, 193-209.

Harris and Vickers (1987, January). Racing with uncertainty. Reviews of Economic Studies $54(1), 1-21$.

Hartman (1960, September). On boundary value problems for systems of ordinary, nonlinear, second order differential equation. Trans.Am.Math.

Hartman, P. (1964). Ordinary Differential Equation. John Wiley.

Horner (1999). A perpetual race to stay ahead: the continous-time analogue. mimeo UPenn.

Moscarini and Smith (2007, November). Optimal dynamic contests. Cowles Foundation Working Paper, Yale University.

Reinganum (1983). Uncertain innovation and the persistence of monopoly. American Economic Review 73, 741-748. 